\title{
Un discours sur prescription : les « argumentaires » des partis politiques comme éléments de cadrage de la parole
}

A Discourse on Prescription: The "argumentaires" of Political Parties as Framing Elements of Speech

\section{Alice Krieg-Planque}

\section{OpenEdition Journals}

Édition électronique

URL : http://journals.openedition.org/aad/1438

DOI : 10.4000/aad.1438

ISSN : 1565-8961

Éditeur

Université de Tel-Aviv

\section{Référence électronique}

Alice Krieg-Planque, « Un discours sur prescription : les « argumentaires » des partis politiques comme éléments de cadrage de la parole ", Argumentation et Analyse du Discours [En ligne], 10 | 2013, mis en ligne le 10 avril 2013, consulté le 23 septembre 2019. URL : http://journals.openedition.org/aad/1438 ; DOI : 10.4000/aad.1438

Ce document a été généré automatiquement le 23 septembre 2019.

\section{cc)}

Argumentation \& analyse du discours est mis à disposition selon les termes de la licence Creative Commons Attribution - Pas d'Utilisation Commerciale - Pas de Modification 4.0 International. 


\title{
Un discours sur prescription : les «argumentaires » des partis politiques comme éléments de cadrage de la parole
}

\author{
A Discourse on Prescription: The "argumentaires" of Political Parties as Framing \\ Elements of Speech
}

Alice Krieg-Planque

\section{Introduction}

1 Nous nous intéressons ici à un genre de texte appelé « argumentaire », tel qu'il existe en tant que production discursive des partis politiques français contemporains. Sommairement, l'« argumentaire » peut être décrit comme un document qui présente notamment un ensemble de positions, généralement argumentées, données à voir comme étant celles de l'organisation partisane sur un thème ou sujet donné. Néanmoins, comme on va le constater, c'est moins le bornage univoque de la définition de ce genre qui fait l'objet de notre investigation que la mise en évidence des principales questions qu'il soulève pour l'analyste du discours.

2 Ainsi, cinq aspects de l'argumentaire retiennent plus particulièrement notre attention. Dans un premier temps, nous mettons en évidence le fait que l'« argumentaire » comme genre existe avant tout par la dénomination dont il fait l'objet de la part des acteurs eux-mêmes. Nous soulignons à cette occasion que la question de l'identification générique ne peut pas être posée indépendamment de celle du métalangage ordinaire. Ensuite, nous étudions certaines des spécificités internes au genre, telles que quelques exemples d'argumentaires des années 2009 à 2011 nous permettent de les repérer. On verra que l'observation la plus marquante réside dans la grande variabilité de l'accueil que les argumentaires font au dialogisme interdiscursif, alternant fermeture du 
discours sur lui-même, dans une visée d'affirmation, et ouverture à l'interdiscours, dans des perspectives de contre-argumentation. Dans la partie suivante, nous mettons l'accent sur l'argumentaire comme document de communication: dans le cadre de la production de discours institutionnels, dont une des finalités est de pérenniser l'institution, les énoncés qui forment les argumentaires apparaissent comme des énoncés conçus pour être repris, assurant ainsi la capacité du parti à "parler d'une seule voix». Dans la subdivision suivante, nous nous intéressons aux différentes finalités et fonctions que les argumentaires sont susceptibles de porter pour le parti politique qui en est le producteur. Sont ainsi évoquées des fonctions d'affirmation de l'autorité du parti, de formation des militants, d'animation du débat public, ou encore de persuasion. Enfin, dans une dernière partie, nous soulignons la situation paradoxale dans laquelle la généralisation de l'internet semble mettre l'argumentaire, qui préserve ses caractéristiques de document destiné aux militants, tout en faisant l'objet d'une large publicisation qui lui confère le statut d'un document de communication externe.

\section{L'« argumentaire » comme dénomination indigène : identification générique et métalangage ordinaire}

\subsection{L'« argumentaire » dans les partis politiques : un type de document identifiable du point de vue de l'organisation}

3 En tant que genre, l'« argumentaire » ne peut pas être dissocié du fait qu'il est identifié comme genre sous ce nom par les acteurs mêmes qui le produisent. En effet, cette dénomination existe dans les appareils des partis politiques, comme l'attestent quatre illustrations que compléterait certainement une enquête sociologique auprès des acteurs.

4 En 2009, le secrétariat national du Parti Socialiste (PS) comportait un pôle « Expression du parti », composé notamment d'un « Porte-parole » et d'un secrétaire à la «Riposte » (en charge de réagir aux prises de position de la droite). Au même moment, le site internet du PS comportait une rubrique «Militant», subdivisée en "Arguments et ripostes » et "Données et arguments ». Cette seconde sous-rubrique donnait accès à une sous-sous-rubrique «Expressions» depuis laquelle il était possible de télécharger des "Argumentaires" (aux côtés de "Communiqués », "Discours ", "Questions d'actu », « Tracts, affiches », "Pétitions »). Les argumentaires en question portaient par exemple sur "L'Europe face à la crise : la relance des socialistes », « Le Pacte européen de progrès social», "Lutter contre le changement climatique» ou "Pour une croissance verte $»^{1}$.

En mai 2009, le site web du Parti Communiste Français (PCF) comportait notamment une rubrique "Tous les tracts ", à partir de laquelle il était possible de télécharger des «Tracts", des "Affiches» et des "Argumentaires» - tel qu'un «Argumentaire pédagogique à l'attention des militant(e)s pour la campagne en vue des élections européennes du 7 juin 2009 », sur lequel nous reviendrons. En 2011, le site web du PCF avait évolué, mais on pouvait toujours y télécharger des argumentaires, tel cet «Argumentaire. Campagne Front uni contre la vie chère " (présent sur la page « Tous les tracts", accessible depuis la sous-rubrique «Matériels» depuis la rubrique «Action »). 
6 En janvier 2009, l'UMP Lycées $^{2}$ diffusait par l'intermédiaire de son site web un document intitulé "Les suppressions de postes dans l'Education nationale ", que nous citerons plus loin, téléchargeable sous le titre «Argumentaire sur les suppressions de postes ».

7 Ou encore, en juillet 2009, le magazine Démocrates, journal interne adressé par le parti centriste MoDem (Mouvement Démocrate) à ses adhérents promouvait l'Université de rentrée organisée par le parti en soulignant le fait que cet événement avait notamment la vocation suivante :

Pour les adhérents et sympathisants, [l'Université vise à] offrir une formation de qualité destinée à leur donner les outils qui les aideront dans les futures campagnes électorales (les valeurs démocrates, élaborer un argumentaire, prise de parole en public, media training...) grâce à la présence de formateurs reconnus ${ }^{3}$.

8 Tous ces exemples attestent l'existence, du point de vue des partis politiques euxmêmes, d'« argumentaires » identifiables sous ce nom.

\subsection{Travailler sur les genres de discours : assumer le métalangage utilisé par les acteurs}

Dans un article consacré à "Types, modes et genres ", Sonia Branca-Rosoff rappelle que « les usagers de la langue classifient spontanément leurs productions discursives » (1999: 5). Elle ajoute :

Par exemple, dans les médias, les journalistes, et leurs lecteurs emploient fait divers, reportage, débats. De même, notes de synthèse, compte rendu... s'entendent dans les bureaux et dans les entreprises; dissertation, thèse, compte rendu de lecture... à l'Université.

Dans le cas présent, "argumentaire » se dit, s'écrit, se lit et s'entend dans les partis politiques. « Argumentaire » présente ainsi deux caractéristiques remarquables. D’une part, il apparaît comme une dénomination relevant du lexique métalinguistique nonsavant (ou non-spécialisé, ou profane, ou ordinaire), c'est-à-dire, dans le cas présent, non-savant du point de vue du linguiste ou du spécialiste des sciences du langage. D'autre part, il relève de ce que Jacqueline Authier-Revuz (1995) appelle un « discours autre approprié ", c'est-à-dire approprié à l'objet dont il parle ${ }^{4}$ : dans le cas présent, le terme métalinguistique "argumentaire » est approprié aux acteurs politiques, en ce sens que ce terme est avant tout celui de ces acteurs. Pour cette raison, nous parlons d'« argumentaire » comme d'une dénomination indigène : produite et utilisée par les acteurs eux-mêmes, cette dénomination générique fonctionne y compris comme catégorie pratique dans le cadre d'ethno-méthodes et de routines de travail permettant aux acteurs d'accomplir leurs tâches - pour emprunter à la perspective de la sociologie pragmatique et de l'ethnométhodologie.

11 Comme c'était le cas dans notre étude sur (les) «petite(s) phrase(s)» (Krieg-Planque 2011), en nous intéressant en tant que scientifique aux " argumentaires", nous nous intéressons ainsi à un objet que l'on désigne par un terme qui est en usage chez les locuteurs eux-mêmes. Le linguiste, l'analyste du discours, le sociologue, le politiste, etc. s'expriment alors avec le langage de leur objet (langage-objet), et se privent d'un métalangage spécifique qui serait extérieur à l'objet étudié (métalangage). Cette situation, en définitive très fréquente dans les travaux sur les "genres de discours ", méritait d'être explicitée. 


\subsection{Des techniques commerciales à l'institution militaire : l'« argumentaire » entre discours promotionnel et expression de la norme}

12 S'il est vrai que "argumentaire " comme dénomination générique relève bien du lexique métalinguistique ordinaire, il n'est pas à cet égard exclusif des partis politiques. En effet, nombreux sont les milieux professionnels et sociaux qui ont recours au terme " argumentaire» pour désigner des types de productions discursives plus ou moins normées. En France, dans l'institution militaire, l'« argumentaire » peut renvoyer à un genre relativement identifiable. C'est ainsi qu'une brochure interne à l'Armée de Terre définit l'« argumentaire ", plutôt destiné à une communication interne, par différence avec l'«élément de langage ", privilégié dans la communication externe. Dans le «Glossaire » qui clôt cette brochure, on peut lire en effet ${ }^{5}$ :

Argumentaire: Ensemble d'informations à propos d'un sujet particulier, le plus souvent présenté sous forme de questions/réponses, permettant à un acteur de se forger une opinion et d'être capable de traiter un sujet avec pertinence. Normalement à usage interne, les argumentaires ne sont cependant pas classifiés et peuvent, exceptionnellement, être transmis en externe pour alimenter le background d'un journaliste.

Elément de langage: Argumentaire thématique à usage externe qui permet de donner la position de l'armée de terre sur un sujet précis. Il existe des éléments de langage génériques et d'autres spécifiques adaptés à une situation particulière.

D'autres organisations utilisent le terme " argumentaire » pour désigner avant tout des documents porteurs de contenus consensuels pour un milieu donné ou pour un ensemble de pratiques professionnelles données. Par exemple, les autorités de santé et agences de sécurité sanitaire (ANSM, InVS...) produisent des documents appelés

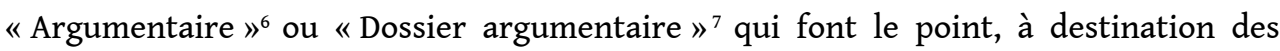
professionnels de santé, sur les données actualisées sur une question, souvent en lien avec ce que les professionnels appellent les « recommandations de bonnes pratiques cliniques ".

On le voit, les "argumentaires » ont un rapport étroit à la norme, au consensus, aux positions communes. Mais certains usages du terme sont moins orientés sur le caractère consensuel des contenus du document que sur sa valeur supposément persuasive. C'est ainsi que les professionnels du marketing et du commerce conçoivent un «argumentaire de vente» ou un "argumentaire commercial»: il s'agit d'inventorier et d'exprimer les caractéristiques du produit ou du service dans le but de valoriser celui-ci auprès d'un potentiel acquéreur.

Cette acception commerciale est, semble-t-il, à l'origine du terme, puisque les dictionnaires historiques de langue française attestent le dérivé « argumentaire » entre 1960 et 1970 dans le domaine de la communication et de la publicité, pour observer sa migration vers le domaine politique en 1977. En effet, d'après le Dictionnaire historique de la langue française (Rey 2010), le dérivé "argumentaire », substantif, " (1960) désigne une série d'arguments publicitaires, puis politiques (1977) ». Selon Le Grand Robert de la langue française (Rey 2001), "argumentaire » apparaît en 1970, dérivé d'« argument ». Dans sa valeur adjectivale, il est utilisé dans le domaine de la communication et de la publicité : "Qui concerne les arguments de vente. "Textes argumentaires. Liste argumentaire" ». Dans sa valeur substantivale, il est utilisé dans le domaine de la 
communication : «Documentation réunissant des arguments de vente. Ensemble de ces arguments. "

Certains usages semblent mêler la visée synthétique (plutôt portée par les administrations et les organisations politiques et publiques) et l'objectif de valorisation (plutôt dominant dans la fonction communicationnelle, publicitaire et commerciale). Ainsi, dans le champ de l'import-export, des investissements à l'étranger ou dans les territoires, les «argumentaires sectoriels ", qui synthétisent les atouts d'un territoire donné pour l'investisseur potentiel dans un secteur donné, semblent opérer la jonction entre le politique et le commercial, sous-tendue par l'idée même de marketing territorial (« attractivité des territoires »...).

\section{Les (ir)régularités internes du genre : variabilité du dialogisme interdiscursif}

\subsection{De l'identification sociale à l'instabilité formelle : la double existence des genres de discours}

Dans l'article déjà cité, Sonia Branca-Rosoff (1999 : 6) observe :

Lorsqu'on adopte un point de vue descriptif et non plus normatif, on s'aperçoit qu'il n'y a pas recouvrement entre la définition sociale des genres (qui catégorise des individus inscrits dans des situations) et le point de vue formel (qui regroupe des productions langagières sur la base de marques linguistiques et de fonctionnements discursifs).

Dans le cas qui nous intéresse, cette remarque s'avère tout à fait pertinente : s'il existe bel et bien une identité sociale du genre (soulignée précédemment à travers la question de la dénomination indigène), le point de vue formel fait apparaître une grande diversité de réalisations (pouvant alors faire douter de l'existence d'un genre, si l'on s'en tenait à un point de vue descriptif). Les observations qui suivent l'indiquent clairement.

\subsection{Taille, structure, graphisme, énonciation... : les irrégularités multiples des argumentaires}

19 Ce n'est certainement pas la longueur du document qui assure la stabilité du genre : en effet, les argumentaires peuvent occuper une page, mais aussi trois ou quatre, et jusqu'à 31 pages pour l'« Argumentaire pédagogique à l'attention des militant(e)s pour la campagne en vue des élections européennes du 7 juin 2009 » [PCF 2009 Européennes].

Les argumentaires présentent également une grande variété quant à leur structuration interne. Tel argumentaire est structuré en opposant sur différents sous-thèmes le «Constat » et les «Propositions » [PCF 2011 Vie chère], quand tel autre est organisé en plan détaillé avec trois niveaux de subdivision numérotés [UMP 2011 Immigration], et un autre encore se donne à voir comme une note, nominativement signée, d'une personne qui s'avère être membre du bureau politique et délégué national aux « études et argumentaires » [FN 2011 Présidentielles]. On est loin, ici, de la régularité observable pour d'autres genres pourtant proches, mais bien plus étroitement normés (tel que le communiqué de presse, par exemple). 
21 Il est à noter qu'à l'intérieur d'une même période temporelle, pour un même parti, les argumentaires peuvent varier sensiblement quant à leur organisation générale. Par exemple, pourtant téléchargeables à partir d'une même page internet, et datés respectivement de mai 2010 et de juin 2010, deux argumentaires du Parti ChrétienDémocrate (PCD) [PCD 2010 Retraites et PCD 2010 Bioéthique] présentent des structures différentes : dans le premier, intitulé " Réforme des retraites. Enjeux et perspectives ", sont présentés successivement en trois parties distinctes la "Situation actuelle», le «Problème ", puis les "Solutions à court terme préconisées par le PCD »; dans le second, sont énumérées six thématiques liées aux «grands enjeux de la bioéthique " («Don de gamète ou d'organe», "Gestation pour autrui »...), chacune étant suivie explicitement de la position du parti formulée à l'aide d'un verbe de parole (« Le PCD rappelle... », «Le PCD constate... », «Le PCD refuse... », «Le PCD demande... »). Une enquête de facture sociologique sur la division du travail de production discursive à l'intérieur des partis politiques et une cartographie des acteurs en charge de la rédaction de ces documents permettraient d'apporter un éclairage supplémentaire sur cette hétérogénéité des réalisations génériques.

Les composantes iconiques et graphiques (typographie, polices de caractères, mise en page, code couleur, logo...) font apparaître la même diversité : le moins que l'on puisse dire, c'est que ce n'est pas sur les argumentaires que portent les plus gros efforts des partis pour imposer une charte graphique.

Certains argumentaires ont manifestement fait l'objet d'un soin maîtrisé et coordonné, et ont été pris dans des circuits de relecture et de validation rigoureux, quand d'autres sont rédigés de façon plus rapide ou moins contrôlée, comme en témoignent alors notamment les fautes de langue et une certaine hétérogénéité stylistique. Là encore, ce n'est pas par son degré d'intégration dans les procédures de validation des documents institutionnels que l'on pourra définir l'argumentaire.

Les modalités d'énonciation sont également variées. Si la modalité allocutive est plutôt rare (et même absente en dehors du paratexte), et si la modalité délocutive semble plutôt privilégiée (le parti parlant alors de lui-même à la troisième personne), la modalité élocutive n'est pas délaissée (au moyen d'un "nous» qui renvoie tendanciellement au parti, tout en rendant possible différentes valeurs inclusives «nous » les Français, « nous » les démocrates...).

De même, si les textes semblent plutôt dominés par des énoncés à caractère descriptif, les accusations plus polémiques ne sont pas totalement à exclure: ainsi du PCF qualifiant la politique du président Nicolas Sarkozy de «marché de dupes» [PCF 2011 Vie chère], ou de l'UMP titrant ironiquement l'un de ses argumentaires "Martine Aubry à Avignon défend la culture... de la démagogie » [UMP 2011 Aubry].

En définitive, il serait bien audacieux de dresser une liste des critères permettant d'attester, par une description formelle interne au document, l'existence d'un « argumentaire » (comme ce serait le cas pour attester l'existence d'un «sonnet » en poésie, par exemple).

\subsection{Le dialogisme interdiscursif, enjeu majeur des argumentaires}

27 En revanche, les argumentaires attirent l'attention par le rapport particulièrement intense qu'ils entretiennent avec les discours qui leur sont extérieurs. En effet, les argumentaires semblent ponctués par des énoncés qui dénient toute existence d'un 
extérieur au discours (posant alors l'argumentaire comme un texte aux bords clos qui asserte avec autorité), et d'énoncés qui, tout à l'inverse, semblent offrir une large place aux discours extérieurs, et adverses, qu'ils convoquent sur des modes divers pour leur opposer réfutations, dénégations, contre-arguments et contradictions. Ainsi, les argumentaires semblent avant tout marqués par un rapport particulièrement tendu à l'extériorité discursive, à l'égard de laquelle ils se montrent alternativement particulièrement fermés ou particulièrement ouverts. Selon ces considérations, la caractéristique générique la plus affirmée des argumentaires est que ceux-ci entretiennent une relation singulièrement aiguë avec le dialogisme interdiscursif, alternativement occulté ou exhibé - l'un et l'autre n'étant pas exclusifs, à l'intérieur d'un même argumentaire.

\subsubsection{Présuppositions et aphorismes : se soustraire à la contradiction} présupposition sont particulièrement remarquables. En effet, les présupposés sont, pour un discours, parmi les moyens les plus frontaux de se présenter comme évident, non-contestable, fermé à la contradiction - c'est-à-dire ne prévoyant pas même cette contradiction. Certains argumentaires ont recours à ce procédé consistant à renvoyer, implicitement, à une proposition qui se trouve soustraite à la contradiction. Ainsi, fidèle à leur doctrine respective, le PCF présuppose qu'il existe une exploitation capitaliste, tandis que le FN présuppose conjointement que les familles démissionnent en matière éducative et que l'immigration massive génère des problèmes d'abaissement de niveau scolaire :

[PCF 2011 Vie chère]

De tous les côtés, c'est le renforcement de l'exploitation capitaliste, pour augmenter la rentabilité des capitaux.

[FN 2011 Présidentielles]

Ne prenant pas en compte à la fois la démission des familles en matière d'éducation et les problèmes d'abaissement du niveau scolaire générés par l'immigration massive, le Projet PS, refusant de traiter la question en amont, propose la multiplication du nombre de classes (plus petites), de professeurs, de "personnel d'accompagnement ", etc.

Sur un autre mode, les énoncés de nature aphoristique participent également de discours qui se donnent à voir comme soustraits à la contradiction. Ainsi retrouve-t-on, dans les énoncés qui suivent, des positions chères au PCF et au PCD, avancées sous la forme d'énoncés de portée générale et universelle :

[PCF 2009 Européennes]

Comme toute institution, l'Union européenne est le produit de la lutte des classes et de la cristallisation à un moment donné des rapports de forces existants.

[PCD 2010 Bioéthique]

Vendre son corps revient à se vendre soi-même, à violer sa propre dignité. ou encore :

Chaque enfant doit pouvoir être accueilli au sein d'un foyer stable composé d'un homme et d'une femme mariés. 


\subsubsection{Négations polémiques et mises en scène dialoguées : représenter la parole de l'autre pour la réfuter}

31 A l'intérieur de la vaste gamme des réponses possibles au dialogisme interdiscursif constitutif de toute parole, d'autres énoncés accordent au contraire une place aux discours extérieurs. Les formes et les modes varient dans la façon d'accueillir l'interdiscours. Dans les argumentaires étudiés, la négation polémique est manifestement un type de formulation prisé. Elle est parfois associée à des formes de représentation du discours autre («comme certains le pensent», «certains rétorqueront que »), et forme alors l'expression la plus caractéristique de la production d'un contre-discours associée à la dénonciation des mensonges de l'adversaire.

[UMP Lycées 2009 Postes]

L'argent des suppressions de postes n'ira pas dans les poches du Président [Sarkozy] comme certains le pensent. Elle servira à financer des stages, à acquérir du nouveau matériel... Bref, elle servira à l'Education nationale. Certains rétorqueront que des lycées manquent de moyens : il s'agit là aussi d'une mauvaise gestion de l'argent qui leur est attribuée, cela ne dépend pas du gouvernement mais du lycée lui-même.

[PCF 2011 Vie chère]

Ce retour de l'inflation n'est pas le fait des salariés puisque la rémunération du travail et des prestations sociales est en recul par rapport à la hausse des dépenses incompressibles (loyers, EDF...). Ce n'est pas non plus le fait des dépenses publiques utiles puisque là encore la part des rémunérations publiques dans le BIP baisse (OCDE). [...] Il y a des bénéficiaires de l'inflation: les marchés, les banques, les spéculateurs [...].

[UMP 2011 Immigration]

La maîtrise de l'immigration n'est pas une obsession franco-française! Tous les Etats du monde - à commencer par les pays du Sud qui font face à des flux très importants de population! - contrôlent leurs frontières et ont une politique d'immigration. C'est une prérogative de base d'un Etat souverain. Avant d'accuser l'UMP de se «droitiser» quand nous voulons maittriser notre politique d'immigration, il faut regarder quelles sont les politiques conduites dans le monde.

32 Toujours dans l'ordre de l'accueil du dialogisme interdiscursif, la mise en scène dialoguée "question - réponse " présente un intérêt certain. En effet, elle permet de donner à voir comme par anticipation les questions ou les objections auxquelles le parti est supposément soumis, et auxquelles il est à même d'apporter des réponses. Dans l'exemple qui suit, la question présente en outre une précieuse ambiguïté du pronom «nous» (où se superposent valeur exclusive - «nous» le PCD, et valeur inclusive «nous » la culture/civilisation européenne/chrétienne):

[PCD 2010 Burqa]

Pourquoi le port de la burqa nous choque-t-il tant?

Parce qu'au cœur de nos valeurs résident la tradition millénaire de la théologie de l'incarnation propre au christianisme, toute une philosophie du visage et de la relation entre les personnes. Nous sommes les héritiers et les praticiens d'une culture selon laquelle la rencontre avec l'autre permet aussi d'expérimenter ce qui, en lui, nous ouvre sur l'absolu. C'est donc une crise culturelle et spirituelle que provoque la burqa.

Si l'on s'en tient strictement à ses réalisations linguistiques formelles, la structure "question - réponse" est plutôt rare. Néanmoins, on relèvera ces paragraphes introductifs de l'argumentaire du PCF, lequel est en réalité organisé en 40 thématiques titrées («Les 27 Etats membres de l'Union Européenne », «L'élargissement et la 
Turquie », "Utilité et rôle du Parlement européen »...), mais que les rédacteurs conçoivent, manifestement, comme un ensemble de " questions » et de " réponses »:

[PCF 2009 Européennes]

Pour mieux maîtriser les enjeux des prochaines élections européennes [...] et pour répondre à une forte demande de compréhension du fonctionnement de l'Europe actuelle, le collectif Europe du Conseil national du PCF a élaboré cet argumentaire sous forme de 40 questions / réponses.

Cet argumentaire vient en complément des textes reprenant notre ambition de changer d'Europe, notre démarche de rassemblement avec le Front de gauche.

D'autres outils sont ou seront à votre disposition comme le bilan de l'action du groupe GUE, [...].

Support pour le débat et l'organisation de réunions publiques, cet argumentaire permet de mener une campagne de proximité pour créer une dynamique populaire, comme nous l'avons réussie en 2005 lors du référendum pour le Traité Constitutionnel Européen.

\subsection{Les textes prescriptifs : une vive sensibilité à la question de l'ouverture-fermeture aux discours extérieurs}

La place manque ici pour exposer d'autres formes, qui sont variées et nombreuses, de mise en scène de la conflictualité discursive. Au terme de ce parcours consistant à repérer différents modes et différents degrés de fermeture-ouverture à l'extériorité discursive, il importe de noter que certains types de formulations se rencontrent fréquemment dans d'autres genres de textes qui ont également un rapport étroit à la norme et à sa reproduction en discours. Nous pensons aux aphorismes, dont Marc Bonhomme a pu repérer l'importance dans le contexte des discours totalitaires (2012). Nous pensons aussi, sur un autre volet, aux textes en "question - réponse ", dont les réalisations jalonnent l'histoire des textes prescriptifs, alternant « ce qu'il faut dire » et " ce qu'il faut faire», depuis certains catéchismes et certaines grammaires («Combien y a-t-il de parties d'oraison? ", «Qui sont-elles ? »... dans les grammaires donatiennes), jusqu'à de plus contemporains argumentaires ("Quand suspecter un TRALI? Comment faire le diagnostic? $\left.»^{8}\right)$ et textes institutionnels variés qui foisonnent dans les organisations publiques et privées. En somme, à travers certains des procédés auxquels il a recours, l'argumentaire des partis politiques rejoint un ensemble plus vaste de textes qui «disent ce qu'il faut dire », qui fournissent tout à la fois les contenus et les formes de l'expression.

\section{Un document de communication : des énoncés conçus pour être repris}

\section{1. "Argumentaires ", " petites phrases ", « slogans", " communiqués de presse "... : des discours de communication}

Par-delà leur diversité et par-delà la faiblesse de la régularité interne au genre, les argumentaires peuvent être appréhendés comme des documents de communication. Dans un travail antérieur, nous avons défini la communication comme « un ensemble de savoir-faire relatifs à l'anticipation des pratiques de reprise, de transformation et de reformulation des énoncés et de leurs contenus" (Krieg-Planque 2010). Ce cadre d'analyse peut être retenu pour l'étude des argumentaires menée ici. Dans le texte cité, 
nous évoquons d'ailleurs les genres susceptibles d'être appréhendés selon une approche discursive de la communication ainsi proposée: nous mentionnons les slogans, les formules, les petites phrases, les communiqués de presse et les argumentaires. A travers cette définition, il ne s'agit pas, bien sûr, d'appréhender la communication sous le seul jour de la transmission ou de la répétition, mais plus subtilement de considérer que, pour une organisation (parti, entreprise, association, syndicat...), les compétences communicationnelles puisent dans la capacité que possède cette organisation à anticiper la façon dont les discours qu'elle produit seront, ou non, repris, mis en circulation, reformulés, transformés (la capacité à ne pas être repris autrement dit à garder une information secrète - constitue donc également une compétence en matière de communication).

\subsection{Détachabilité, sur-assertion, structuration de l'échange : préparer les discours à leur reprise}

Dans le cas spécifique des argumentaires, la subdivision précédente a permis de repérer certaines formulations particulièrement favorables à la reprise. Les aphorismes, par leurs contenus généralisables et leurs expressions formelles souvent remarquables, sont bien entendu sujets aux phénomènes de détachement énonciatif, suivant les propositions de Dominique Maingueneau (2012). Il en est de même de certaines injonctions, qui semblent prêtes à participer à la création de slogans, comme pourrait l'être ici « agir maintenant pour demain », à propos de l'urgence à réformer le système des retraites :

[PCD 2010 Retraites]

Il est urgent de rendre justice à l'effort des mères et des familles dans le renouvellement des générations, sans quoi la survie du système sera impossible à moyen et long termes. Il faut agir maintenant pour demain.

D'autres types d'énoncés semblent également conçus pour servir à la reprise dans le cadre de documents écrits ou d'échanges oraux. C'est le cas des chiffres, des nombres, des pourcentages, et plus globalement des données chiffrées, dont certains argumentaires sont nourris, et dont la valorisation est parfois assurée, dans le texte, par un soulignement, une mise en gras ou l'italique :

[PCF 2011 Vie chère]

- La dépense moyenne engagée par les ménages pour se loger a augmenté de $23 \%$ entre 2002 et 2007.

- 3.5 millions de femmes et d'hommes vivent dans des logements indignes ou sont sans-abris.

- $80 \%$ des français estiment qu'il est aujourd'hui difficile de trouver un logement.

- Il y a, fin de l'année dernière, 1230136 demandes de logements sociaux sans réponses. La crise du logement touche d'une manière ou d'une autre 10 millions de personnes en France et se traduit par une hausse des loyers. Le coût du logement, dans le secteur privé, représente pour les familles les plus modestes jusqu'à $50 \% \mathrm{du}$ revenu.

- Seuls 32 communes en 10 ans ont atteint le taux de $20 \%$ de logements sociaux sur 931 communes concernées par la loi.

[UMP 2011 Immigration]

II. La politique d'immigration choisie menée par la majorité a déjà fait ses preuves

- La lutte active contre l'immigration illégale depuis 2007 a porté ses fruits :

$>$ Plus de 110000 personnes éloignées vers leur pays d'origine contre 7000 
personnes par an en moyenne entre 1997 et 2002.

> Plus de 100000 personnes refoulées aux frontières avant l'entrée sur notre territoire.

> En 2010, 183 filières ont été démantelées contre 145 en 2009 et 101 en 2008.

C'est le cas également d'une variété d'énoncés qui, sur des modes divers, fournissent au lecteur, futur locuteur potentiel, les moyens de tenir un discours consistant et structuré. Par exemple, les quatre argumentaires proposés par le PS en 2009 dans le cadre de la campagne pour les élections au Parlement européen sont, chacun, structurés en un plan susceptible d'être mobilisé dans le cadre d'une discussion, selon un modèle général « description de la situation et enjeux - attaque de l'adversaire proposition du PS». Sont présentés ci-dessous le titre de l'argumentaire et le premier niveau de subdivision:

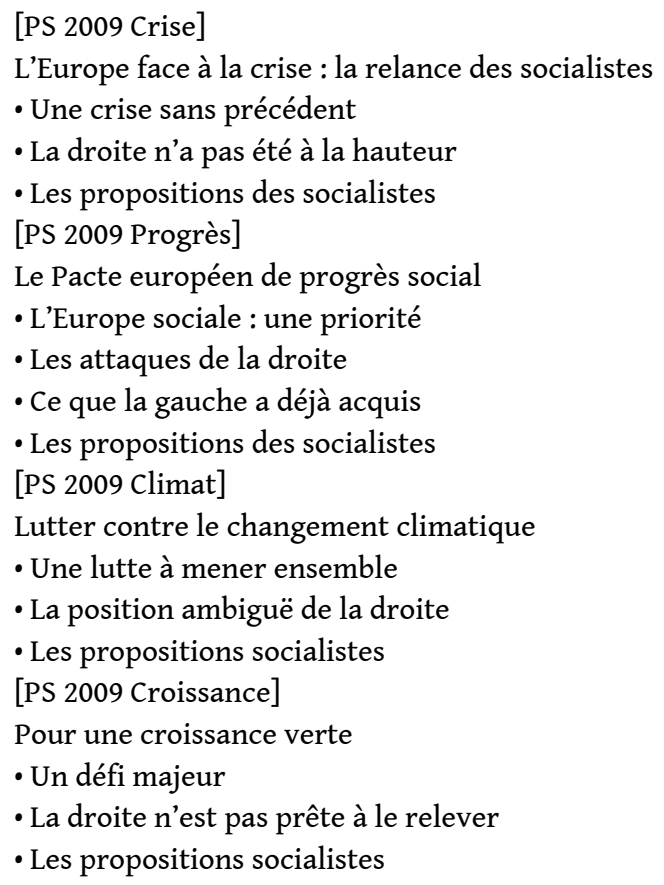

Dans certains argumentaires, la mise en texte et la mise en page elles-mêmes, structurées à travers des listes et des énumérations (tirets, puces, flèches...), participent d'une préparation du discours pour la reprise. Les argumentaires, alors, semblent tout entiers caractérisés par la sur-assertion, entendue comme le marquage dans un texte d'un énoncé comme détachable.

\subsection{L'énonciation institutionnelle, garante des énoncés à faire circuler}

Dans l'étude de l'argumentaire en tant que genre, il importe de souligner le caractère fortement institutionnel du locuteur considéré tout autant que du discours qu'il tient. $\mathrm{Si}$, ainsi que nous l'avons suggéré, les scripteurs des argumentaires sont sans doute très hétérogènes en compétences et en statuts, et si les procédures de validation par l'appareil du parti semblent exercer un contrôle extrêmement variable sur les documents, il n'en reste pas moins que les argumentaires se présentent énonciativement comme des textes pris en charge par le parti lui-même, en tant qu'organisation partisane. Il est extrêmement rare, d'ailleurs, que l'argumentaire soit nominativement signé (au fil de nos recherches, seul le FN est concerné, ainsi que 
quelques argumentaires de l'UMP Lycées), et il est rare également qu'il soit présenté dans le paratexte comme rédigé par une commission, un secrétariat, ou un collectif (seul l'argumentaire du PCF de 2009 est concerné). Ainsi, c'est une légitimité institutionnelle forte qui s'attache à l'argumentaire, en tant que production discursive de l'organisation partisane. Quant au texte lui-même, il est manifeste qu'il entend exprimer et faire connaître la position officielle du parti. L'argumentaire ne se donne pas à voir comme un ensemble de positions acceptables, et moins encore comme un document qui exprimerait les différentes sensibilités du parti, ses courants, ses luttes internes, les apports des personnalités ou des voix dissonantes (alors même que dans d'autres contextes l'existence de différentes sensibilités au sein du parti peut être valorisée, du moins rhétoriquement).

\subsection{Parler d'une seule voix : rendre partageable un « dire conforme »} bien identifiées à propos des discours institutionnels : dit sommairement, ces discours se manifestent par divers types de déni du conflit, l'évitement de la contradiction interne, la mise en avant de la cohérence et de la cohésion, la réduction des dissonances, l'euphémisation des désaccords, des rapports surveillés à la norme et à la prescription. En ce sens, Claire Oger a pu pointer certaines des pratiques discursives de l'institution en tant qu'elles relèvent du «contrôle de la parole » (Oger 2003) ou de procédés de "lissage " (Oger et Ollivier-Yaniv 2006), et nous avons pu identifier un double principe de formation des discours institutionnels, conjuguant stabilisation des énoncés et effacement de la conflictualité (Krieg-Planque et Oger 2010). A certains égards, il est clair que les argumentaires des partis politiques participent de ce vaste ensemble des discours qui visent l'univocité.

42 fait que, tout en portant la position du parti, ils assurent une mise en œuvre qui aide à la rendre reprenable. Il ne s'agit pas seulement d'exprimer et de faire savoir une position, mais de la rendre partageable et diffusable, argumentable, explicable et justifiable. Ceci distingue les argumentaires des résolutions adoptées lors des congrès, par exemple, ou d'autres types de documents qui scandent le rythme de la vie du parti et lui donnent l'occasion d'exprimer discursivement sa cohérence doctrinale et son programme. C'est ainsi que nous appréhendons les argumentaires comme des documents qui, tout en étant fortement marqués par leur appartenance au discours institutionnel, sont en même temps très caractérisés par leur identité de documents de communication. A travers la mise en forme des discours du parti dans ce genre spécifique qu'est l'argumentaire, il s'agit de proposer au lecteur un ensemble de contenus dont la reprise est supposée être facilitée à la fois par la fourniture d'une expression qui porte ces contenus et d'arguments qui soient susceptibles d'en assurer la pédagogie.

Dans son étude des discours de l'institution militaire, Claire Oger repérait que certaines formes de circularité observables dans les «éléments de langage » (par exemple, le fait de reprendre des citations de représentants de l'Armée telles que figurant dans des interviews de la presse) répondait à l'« impératif majeur de la communication des armées, toujours défini comme une priorité : la cohérence du discours " (Oger 2003 : 83). Même si l'institution militaire et les partis politiques constituent des types de 
locuteurs bien différents, les argumentaires que nous avons étudiés présentent une visée analogue de cohérence et de contrôle de la parole : il s'agit d'aider à la diffusion des standards du discours, de garantir au moins la possibilité d'un « dire conforme », de minimiser les risques d'écart relativement à la norme. En même temps (et ceci spécifie certainement les partis politiques, tout en distinguant les « éléments de langage » des " argumentaires »), il s'agit de rendre appropriable le discours du parti, d'en assurer la reprise auprès de locuteurs dont les motivations et les compétences, tant politiques que communicationnelles, peuvent être extrêmement hétérogènes. Une réflexion sur la fonction des argumentaires aide à appréhender cette complexité du destin de l'« argumentaire » comme genre de discours.

\section{Affirmer, former, débattre, convaincre : la multifonctionnalité des argumentaires}

\subsection{Les textes politiques : un réseau complexe d'usages et de fonctions}

Dans le domaine politique comme ailleurs, un document s'inscrit toujours dans un riche réseau d'usages et de fonctions. Le genre «tract ", par exemple, qui a fait l'objet d'études inaugurales en analyse du discours politique (Demontet et al. 1975), et dont on pourrait penser qu'il vise d'abord et avant tout à convaincre (de voter pour tel candidat, de participer à telle manifestation, etc.) peut s'inscrire dans la réalité complexe de ce qu'est une « campagne $»^{9}$, pour laquelle les militants sont, du point de vue de l'appareil du parti, tout autant des ressources auxiliaires à mobiliser que des forces à canaliser.

Selon les moments de la vie politique dans laquelle ils interviennent, selon les publics qui ont recours à eux, ou encore selon que l'on se place du point de vue du parti, du dirigeant ou de l'élu, de l'adhérent, du militant, du sympathisant ou du citoyen peu politisé, voire de l'adversaire ou de l'opposant, les argumentaires ont bien entendu des emplois et des fonctions diversifiées. Quatre d'entre elles peuvent être plus particulièrement soulignées : l'affirmation du parti comme institution, la formation politique et rhétorique du militant, l'animation de la vie politique et du débat public, la persuasion des citoyens.

\subsection{Affirmer la légitimité et l'autorité du parti}

En tant que genre constitutif du discours d'appareil, et aux côtés d'autres genres (résolutions de congrès, programmes, communiqués...), l'argumentaire participe d'une fonction d'affirmation de l'institution. S'autorisant de lui-même et de sa capacité à verbaliser ses propres positions, le parti asserte des thèses et en fixe les formulations légitimes. Ainsi que nous l'avons vu, il lui arrive également de réfuter les thèses adverses, dont il détermine lui-même l'expression (par exemple, l'UMP Lycée donne à voir certains de ses adversaires comme étant susceptibles d'asserter que «des lycées manquent de moyens »).

Par ailleurs, à travers l'établissement de certaines séries thématiques au sujet desquelles des argumentaires sont produits, le parti donne, en discours, le gage de sa capacité à tenir une position: en garantissant, par le moyen des argumentaires, 
l'existence d'une opinion sur le sujet, le parti coupe court à ceux qui voudraient prétendre qu'il n'a « rien à dire » sur le dossier.

Dans son ouvrage consacré aux partis politiques, Michel offerlé souligne qu'à certains égards « les partis ne sont qu'une des formes historiquement déterminées d'entreprise politique " (2012: 12-13). A travers la production d'argumentaires, le parti assoit la légitimité d'une telle forme historique à accomplir une telle activité de production de biens politiques : il se donne à voir comme capable d'en produire les contenus et d'en arrêter les expressions publicisables.

\subsection{Contribuer à la formation des militants}

En second lieu, les argumentaires participent à la formation des militants, aux côtés d'autres dispositifs (réunions de section...) et de divers documents (ouvrages, fascicules, presse interne...). Ils relèvent, sous une forme très spécifique, de ces «lectures militantes» dont un récent numéro de revue a relevé l'importance pour le renforcement d'une culture commune (Ponsard et al. 2009). Dans une perspective de communication interne qui distingue clairement l'«argumentaire " du "tract» ou encore de l'« élément de langage ", il s'agit ici de construire un univers de référence à l'échelle de militants qui disposent nécessairement de compétences hétérogènes, de nourrir la socialisation partisane et de concourir à la formation d'un esprit de parti. Du point de vue de la capacité des militants à se faire les vecteurs de la norme, il s'agit d'enrichir les discours en formes et en expressions autorisées.

Dans le journal des adhérents du MoDem déjà cité, la maîtrise du genre «argumentaire» participait manifestement de l'acquisition d'une compétence communicationnelle de la part des militants. D'autres éléments encore concourent à identifier l'argumentaire comme étant un genre avant tout interne (destiné aux militants) et tourné vers l'action - relevant (on le verra) notamment d'une fonction de persuasion -, dans la perspective d'une amélioration de leur compétence. L'argumentaire du PCF autour des européennes [PCF 2009 Européennes] est adressé, dans son titre, "à l'attention des militants ", et le déterminant possessif renvoyant à l'allocutaire («D'autres outils sont ou seront à votre disposition [...]») réfère bien à l'acteur de la base en action. Quant aux argumentaires du PS de la même période, on rappellera qu'ils étaient téléchargeables depuis une arborescence dont la tête était «Militant ». Deux ans plus tard, l'argumentaire élaboré par le PCF dans le cadre de la campagne "Front uni contre la vie chère » est précédé, dans sa version téléchargeable en pdf, par une lettre qui s'ouvre par la formule «Cher-e Camarade, ", et qui précise notamment: "Cette campagne va prendre appui sur des tracts, une pétition, des affiches (à venir) et cet argumentaire pour les militants. » Il apparaît ainsi clairement que le document s'intègre dans un plus vaste ensemble de matériel militant, dont il constitue l'un des volets internes, comme le précise bien l'expression «pour les militants ", par différence avec les tracts ou les affiches.

51 En dehors du paratexte, et du point de vue des contenus des argumentaires eux-mêmes, la fourniture de données chiffrées, précédemment signalée, et plus globalement d'éléments factuels, semble particulièrement adaptée à cette fonction de formation, par apport de connaissances - dont le militant pourra se servir dans un travail d'argumentation. Par exemple, dans l'argumentaire du PCF produit pour les Européennes [PCF 2009 Européennes], la seconde des 40 « questions - réponses » est : 
2. Les 27 Etats membres de l'Union européenne

Allemagne, Autriche, Belgique, Bulgarie, Chypre, Danemark, Espagne, Estonie, Finlande, France, Grèce, Hongrie, Irlande, Italie, Lettonie, Lituanie, Luxembourg, Malte, Pays-Bas, Pologne, Portugal, République tchèque, Roumanie, Royaume-Uni, Slovaquie, Slovénie, Suède. ces débats, à travers l'organisation de réunions publiques, de soirées débats, etc. Dans l'argumentaire du PCF pour les élections européennes de 2009, un passage déjà cité évoquait ainsi l'utilité du document "pour le débat et l'organisation de réunions publiques». Les argumentaires, ici, jouent tout leur rôle sinon de «cadrage» de la parole, du moins de «guidage » de cette parole. Conçus comme des stocks d'arguments, des matrices de vocabulaire et des structures de l'échange, les argumentaires permettent au militant d'organiser la parole sous le contrôle de l'institution, aux côtés de différents supports relevant de ce que certains partis appellent le "kit militant " (comprenant également des powerpoints, des vidéos, etc.).

de l'institution militaire, Claire Oger $(2003: 81)$ relève que «les argumentaires fonctionnent en premier lieu comme réservoir de vocabulaire ». De façon convergente, nous pouvons estimer que les argumentaires des partis politiques ont notamment pour finalité de participer à la stabilisation de l'expression de locuteurs qui, s'autorisant du parti, vont porter dans la sphère publique un certain nombre de dires autorisés, à travers des formats qui relèvent plus du débat et de l'échange que de la prise de position solennelle et officielle. 


\subsection{Etre un support de persuasion dans l'échange}

instrument aidant à emporter la conviction dans l'échange, dans le cadre de son réemploi par le militant. Dans la continuité de l'acception initiale du terme, qui en faisait un outil de persuasion commerciale, l'argumentaire agit alors comme un stock dans lequel le militant va pouvoir puiser les ingrédients qui lui permettront de produire un discours convaincant. politiques sont des lieux où s'échangent des produits politiques contre des soutiens (matériels, symboliques) et des votes ». Selon cette conception, et dans sa fonction de conviction, l'argumentaire peut être appréhendé comme l'un de ces lieux où s'effectue une telle transaction : l'argumentaire constituerait le support d'expression des produits politiques proposés par le parti, échangés contre un vote dans l'hypothèse d'une réussite du travail de conviction mené par le militant, institué en opérateur sur ce marché. Par ailleurs, en tant qu'il contribuerait à un travail de conviction auprès des citoyens prenant part aux scrutins, l'argumentaire participerait à construire ce que l'on peut considérer comme la « légitimité par les électeurs » du parti politique.

L'accroissement de la production d'argumentaires au moment de scrutins (élections, référendums...), et l'existence même de l'expression "argumentaire de campagne », peuvent sembler conforter la fonction de conviction que les argumentaires seraient susceptibles de remplir. Il en est de même de la mise en place, à ces mêmes moments de la vie politique, de cellules, de groupes et de structures fonctionnelles consacrés aux argumentaires à l'intérieur des partis. En janvier 2011, dans la perspective des élections présidentielles de 2012, le Parti Socialiste alors dans l'opposition se dote d'un groupe appelé "Communication et riposte à la droite», chargé de coordonner les argumentaires pour répondre à la majorité présidentielle. Une telle institutionnalisation de la production d'argumentaires atteste, à certains égards, de la croyance en la possibilité de ce type de document à assurer, dans le cadre d'une communication cohérente, un travail de persuasion.

61 Pour notre part, néanmoins, dans la suite du travail de Marc Angenot exposé dans Dialogue de sourds (2008), on ne saurait trop souligner qu'il n'est pas certain que le déploiement d'une argumentation permette de convaincre. Se persuadant rarement, souligne Angenot, les hommes persévèrent étrangement à argumenter. C'est dans une même interrogation sur les fonctions réellement persuasives de la rhétorique que nous pouvons inscrire notre réflexion, renvoyant alors les argumentaires à d'autres fonctions privilégiées développées plus haut : celles de la légitimation de l'institution et de la formation des militants.

Bien entendu, aucune de ces fonctions n'est exclusive des autres, et il faut plutôt considérer que les argumentaires sont susceptibles d'accomplir l'ensemble de ces fonctions. Pour conclure, à travers une dernière subdivision, c'est moins sur les fonctionnalités elles-mêmes que sur leurs déplacements que nous souhaitons insister. En effet, comme toute analyse générique, l'étude des " argumentaires » doit être située dans le cadre de l'historicité des supports et des évolutions des dispositifs sociotechniques.

Argumentation et Analyse du Discours, 10 | 2013 


\section{Les argumentaires en ligne: lorsque le genre textuel est troublé par le web}

\subsection{L'internet, facteur de changements dans l'organisation et la communication des partis politiques}

Différents chercheurs ont souligné les changements que l'internet (listes de diffusion, puis sites web et réseaux sociaux) a produits et continue de produire sur la vie des partis politiques. Qu'ils s'inscrivent en sociologie politique, en science politique ou en sciences de l'information et de la communication, tous ces travaux insistent sur la diversité des déplacements de frontières et d'organisation que l'internet produit. Dans un travail pionnier qui soumettait à l'analyse les sites web de la période 2004-05, Gersende Blanchard (2007) mettait en évidence une certaine évolution de la communication partisane, caractérisée par une diversification de ses acteurs: elle observait que les sites permettaient à la fois la reproduction de contenus existants (à travers leur fonction d'autopublication, autorisant la mise en ligne de tracts, d'allocution...) et l'autonomisation de la diffusion des discours (à travers la publicisation de paroles d'internautes). Cette seconde dimension des possibilités offertes par l'internet, par laquelle il est possible de "parler politique en ligne ", a été abordée par exemple dans un numéro de Réseaux consacré au sujet (Greffet et Wojcik éds 2007) : internet y apparaît comme un lieu où s'exercent, sur fond d'un idéal délibératif, de nouvelles pratiques argumentatives. Dans un ouvrage coordonné par Fabienne Greffet (2011) et consacré à la façon dont divers partis politiques se sont approprié l'internet, les contributeurs mettent en évidence des usages différenciés : il a pu s'agir avant tout de permettre à certains militants de gagner des positions au sein du parti, grâce à la détention d'une compétence technique (UMP), de contourner des médias traditionnels perçus comme des censeurs (FN), ou encore de chercher à rallier des militants que les anciennes modalités de recrutement ne permettaient pas d'atteindre (PS). Dans ce dernier cas, les usages d'internet par le parti ont donné lieu à des recompositions de la notion d'" adhérent", et à une modification de la relation entre l'adhérent et le parti, comme l'ont mis en évidence Eric Treille et Thierry Barboni (2010). En définitive, c'est à tous égards une reconfiguration des partis et de leur organisation, ainsi que de la conception de la prise de parole politique dans la sphère publique, que l'internet produit.

\subsection{L'argumentaire, un « genre du papier » saisi dans les usages du numérique}

Pour ce qui concerne les argumentaires, internet semble jouer un rôle qui aboutit à une situation paradoxale. A certains égards, l'argumentaire demeure un genre (certes hétérogène) ancré dans l'époque qui l'a vu naître, un "genre du papier » et un genre destiné à la communication interne à destination des militants. A d'autres égards, l'argumentaire est, de fait, pris dans des dispositifs de mise en ligne qui en accroissent la circulation et en diversifient les possibilités d'usages.

Le site web du PCD, parmi d'autres, illustre cette situation. Dès la page d'accueil du site, un bouton très visible s'intitule "Découvrez les argumentaires du PCD. Agriculture, bioéthique, retraites, burqa »[consulté en août 2011]. Ce dispositif, par lequel un 
bouton en page d'accueil donne un accès direct à la liste des argumentaires immédiatement téléchargeables, fait plutôt des argumentaires un genre de la communication institutionnelle: au même titre que peuvent l'être des brochures ou des plaquettes, les argumentaires permettent alors de disposer d'une connaissance générale des positions du parti, à destination de l'internaute ordinaire ou du citoyen curieux. Mais cette même liste d'argumentaires est également accessible par un chemin qui semble plutôt destiné au militant, même s'il n'est pas soumis à une restriction d'accès et demeure facile à trouver : depuis la page d'accueil, à partir de la rubrique "Passer à l'action", il est possible de cliquer sur une sous-rubrique "Ressources", laquelle donne accès à six types de ressources : "Les discours ", "Les argumentaires ", «La lettre d'information», "Les documents», "Les affiches", "Les vidéos». La ressource «Les argumentaires » donne accès à la même URL que le bouton « Découvrez les argumentaires du PCD ». L'argumentaire se trouve ainsi pris dans un double chemin de consultation, qui fait de lui un document dont les usages potentiels sont hybridés.

De fait, d'un certain point de vue, l'argumentaire est bien un "genre du papier ». Né dans les organisations politiques avant la démocratisation de l'internet et avant l'intégration du web dans les stratégies communicationnelles des partis politiques, l'argumentaire conserve les marques formelles les plus caractéristiques de son époque d'origine. Sur les sites web des partis, l'argumentaire existe toujours sous la forme d'un document téléchargeable et imprimable au format A4. Et, du point de vue de son organisation interne, il ne semble pas affecté par les potentialités offertes par l'internet: il ne comporte ni liens hypertextes ni possibilités techniques de navigation vers d'autres documents, et moins encore des formulaires et des possibilités de contribution caractéristiques du « web 2.0 ». Dit autrement, en tant que document doté d'une certaine structure interne, l'argumentaire semble essentiellement imperméable à l'internet. Il est, selon ces considérations, aux antipodes de genres qui ont précisément été conçus sur la base des ressources offertes par l'internet (forums de discussion, blogs, micro-blogging...), et n'a recours à l'internet que pour ses possibilités d'autopublication.

Mais, d'un autre point de vue, par le fait même qu'il fait l'objet d'une mise en visibilité large et publique de la part des partis, l'argumentaire bénéficie de la diffusion que rend possible l'internet. Le site web du PCD illustre bien cette tension dans laquelle est pris chacun des argumentaires de ce parti : à la fois préservé dans sa place historique de document militant et propulsé comme document de communication institutionnelle exposé au public. Dès lors, dans ces déplacements de l'accessibilité du texte, et, partant, des destinataires et des publics, une des interrogations porte sur la façon dont - et auprès de qui - l'argumentaire est désormais supposé opérer, ou non, comme élément de cadrage de la parole. 


\section{BIBLIOGRAPHIE}

Agrikolansky, Eric, Jérôme Heurtaux \& Brigitte Le Grignou (éds). 2011. Paris en campagne. Les élections municipales de mars 2008 dans deux arrondissements parisiens (Broissieux : Editions du Croquant)

Angenot, Marc. 2008. Dialogue de sourds. Traité de rhétorique antilogique (Paris : Mille et Une Nuits)

Authier-Revuz, Jacqueline. 1995. Ces mots qui ne vont pas de soi. Boucles réflexives et non-coïncidences du dire (Paris, Larousse)

Blanchard, Gersende. 2007. La communication politique partisane sur Internet : des pratiques et des stratégies nouvelles? (Thèse de doctorat en Sciences de l'information et de la communication, Université Stendhal - Grenoble 3, soutenue le 26 novembre 2007)

Bonhomme, Marc. 2012. «Rhétorique de l'aphorisme et discours totalitaire ", dans Aubry, Laurence \& Béatrice Turpin (éds). Avec Victor Klemperer, repenser le langage totalitaire (Paris : CNRS Editions)

Branca-Rosoff, Sonia. 1999. « Types, modes et genres : entre langue et discours ", Langage et Société 87 : 5-24

Demontet, Michel, Annie Geffroy, Jean Gouazé, Pierre Lafon \& Maurice Tournier. 1975. Des tracts en mai 1968. Mesures de vocabulaire et de contenu (Paris : Editions de la Fondation nationale des sciences politiques)

Greffet, Fabienne (éd.). 2011. Continuerlalutte.com. Les partis politiques sur le web (Paris : Presses de Sciences Po)

Greffet, Fabienne \& Stéphanie Wojcik (éds). 2008. « Parler politique en ligne », Réseaux 150.

Krieg-Planque, Alice. 2010. « Pour une analyse discursive de la communication : la communication comme anticipation des pratiques de reprise et de transformation des énoncés ", Burger, Marcel, Jérôme Jacquin \& Raphaël Micheli (éds). 2010. Les médias et le politique. Actes du colloque « Le français parlé dans les médias » - Lausanne, 1-4 septembre 2009, Lausanne : Centre de linguistique et des sciences du langage. [En ligne : http://www.unil.ch/clsl/page81503.html].

Krieg-Planque, Alice. 2011. «Les "petites phrases" : un objet pour l'analyse des discours politiques et médiatiques ", Communication \& Langages $168: 23-41$.

Krieg-Planque, Alice \& Claire Oger. 2010. «Discours institutionnels : perspectives pour les sciences de la communication », Mots $94:$ 91-96.

Maingueneau, Dominique. 2012. Les phrases sans texte (Paris : Armand Colin).

Nicourd, Sandrine (dir.). 2009. Le travail militant (Rennes : Presses Universitaires de Rennes).

Offerlé, Michel. 2012. Les partis politiques (Paris : Presses Universitaires de France)

Oger, Claire. 2003. «Communication et contrôle de la parole : de la clôture à la mise en scène de l'institution militaire », Quaderni. La revue de la communication 52 : 77-92.

Oger, Claire \& Caroline Ollivier-Yaniv. 2006. « Conjurer le désordre discursif. Les procédés de 'lissage' dans la fabrication du discours institutionnel », Mots 81 : 63-77.

Ponsard, Nathalie (et al.). 2009. « Lectures militantes au XXème siècle », Siècles. Cahiers du Centre d'histoire Espaces et cultures 29. 
Rey, Alain (éd.). 2001 rééd. Le Grand Robert de la langue française (Paris : Editions Le Robert).

Rey, Alain (éd.). 2010 rééd. Dictionnaire historique de la langue française (Paris : Editions Le Robert).

Treille, Eric \& Thierry Barboni. 2010. «L'engagement 2.0. Les nouveaux liens militants au sein de l'e-parti socialiste », Revue Française de Science Politique 60-6 : 1137-1157.

\section{ANNEXES}

Liste des argumentaires cités :

[PCF 2009 Européennes] « Argumentaire pédagogique à l'attention des militant(e)s pour la campagne en vue des élections européennes du 7 juin 2009 », Parti Communiste Français, avril 2009. Téléchargé en mai 2009 depuis le site web du PCF (www.pcf.fr), accueil > « Tous les tracts ».

[PCF 2011 Vie chère] « Argumentaire. Campagne Front uni contre la vie chère ", Parti Communiste Français, mai 2011. Téléchargé en août 2011 depuis le site web du PCF (www.pcf.fr), accueil > « Action » > « Matériels » > « Tous les tracts ».

[PS 2009 Climat] « Lutter contre le changement climatique », Parti Socialiste, 2009. Téléchargé en juin 2009 depuis le site web du PS (www.parti-socialiste.fr), accueil > « Militant » > « Données et arguments » > « Expressions » > « Argumentaires ».

[PS 2009 Crise] «L'Europe face à la crise : la relance des socialistes », Parti Socialiste, 2009. Téléchargé en juin 2009 depuis le site web du PS (www.parti-socialiste.fr), accueil > « Militant » > « Données et arguments » > « Expressions » > « Argumentaires ».

[PS 2009 Croissance] « Pour une croissance verte », Parti Socialiste, 2009. Téléchargé en juin 2009 depuis le site web du PS (www.parti-socialiste.fr), accueil > « Militant » > « Données et arguments » > « Expressions » > « Argumentaires ».

[PS 2009 Progrès] « Le Pacte européen de progrès social », Parti Socialiste, 2009. Téléchargé en juin 2009 depuis le site web du PS (www.parti-socialiste.fr), accueil > « Militant » > « Données et arguments » > « Expressions » > « Argumentaires ».

[UMP 2011 Immigration] « Répondre aux défis de l'immigration », Union pour un Mouvement Populaire, juillet 2011. Téléchargé en août 2011 depuis le site de l'UMP (www.lemouvementpopulaire.fr), accueil > « Retrouvez tous nos argumentaires ».

[UMP 2011 Aubry] « Martine Aubry à Avignon défend la culture... de la démagogie », Union pour un Mouvement Populaire, juillet 2011. Téléchargé en août 2011 depuis le site de l'UMP (www.lemouvementpopulaire.fr), accueil > « Retrouvez tous nos argumentaires $»$.

[UMP Lycées 2009 Postes] « Les suppressions de postes dans l'Education nationale », Union pour un Mouvement Populaire Lycées, janvier 2009, signé Willy Delaunay. Téléchargé en février 2009 sous le titre «Argumentaire sur les suppressions de postes » depuis le site web de l'UMP Lycées (www.ump-lycees.fr).

[PCD 2010 Retraites] « Réforme des retraites, enjeux et perspectives », Parti ChrétienDémocrate, mai 2010. Téléchargé en août 2011 depuis le site web du PCD (www.partichretiendemocrate.fr), accueil > « Passer à l'action » > « Ressources » > « Les argumentaires ». 
[PCD 2010 Bioéthique] « Les grands enjeux bioéthiques », Parti Chrétien-Démocrate, juin 2010. Téléchargé en août 2011 depuis le site web du PCD

(www.partichretiendemocrate.fr), accueil > « Passer à l'action » > « Ressources » > « Les argumentaires ».

[PCD 2010 Burqa] « Réponses au problème de la burqa en France », Parti ChrétienDémocrate, avril 2010. Téléchargé en août 2011 depuis le site web du PCD (www.partichretiendemocrate.fr), accueil > « Passer à l'action » > « Ressources » > « Les argumentaires".

[FN 2011 Présidentielles] « Analyse des propositions du PS pour la présidentielle de 2012 », Front National, mai 2011, signé Thibaut de La Tocnaye. Téléchargé en août 2011 depuis le site du FN (www.frontnational.com), accueil > « Nos idées » > « Les argumentaires".

\section{NOTES}

1. La liste des argumentaires cités dans ce travail figure en fin de texte.

2. L'UMP Lycées est une organisation lycéenne intégrée au mouvement des Jeunes Populaires, lequel est la branche jeune de l'Union pour un Mouvement Populaire (UMP).

3. MoDem, Démocrates. Le journal du mouvement démocrate, $\mathrm{n}^{\circ} 3$, juillet 2009 , p. 8 , rubrique « Vie du mouvement », « Université de rentrée 2009 ».

4. Dans l'analyse du métadiscours, et plus spécifiquement dans l'analyse de la représentation du discours autre, Jacqueline Authier-Revuz (1995) propose de distinguer le «discours autre approprié », c'est-à-dire approprié à l'objet dont il parle ( Il est en train de "draguer " comme il dit») et le «discours autre associé», c'est-à-dire associé pour une raison ou pour une autre à l'objet dont il parle ("Il est en train de lui "conter fleurette" comme disait ma grand-mère »).

5. Communiquer avec les médias. Le guide, SIRPA Terre, Paris, Ministère de la Défense, 2002, p. 39 et p. 40. Pour une analyse des «argumentaires » et des « éléments de langage » dans le contexte de l'institution miliaire, voir Oger (2003).

6. Voir par exemple: «Mise au point sur le TRALI. Argumentaire », Afssaps, 17 juillet 2006, consultable sur www.afssaps.fr/content/download/20953/.../map-Trali_argumentaire.pdf

7. Voir par exemple : « Réévaluation des pénicillines du groupe $M$ administrées par voies orale et injectable : Oxacilline et Cloxacilline. Dossier Argumentaire ", Afssaps, mai 2011, consultable sur http://www.infectiologie.com/site/medias/_documents/officiels/afssaps/2011-peni_M-

AFSSAPS-argumentaire.pdf

8. «Mise au point sur le TRALI. Argumentaire », Afssaps, op. cit.

9. Sur la «campagne», voir le récent travail mené en sociologie politique par un collectif de chercheurs dans le contexte d'élections locales : Agrikolansky, Heurtaux, Le Grignou dir. (2011).

\section{RÉSUMÉS}

Nous nous intéressons à un genre de texte, appelé " argumentaire ", tel qu'il existe en tant que production discursive des partis politiques français contemporains. Sommairement, 
l'«argumentaire » peut être décrit comme un document qui présente notamment un ensemble de positions, généralement argumentées, données à voir comme étant celles de l'organisation partisane sur un thème ou sujet donné. Cinq aspects de l'«argumentaire » retiennent plus particulièrement notre attention: sa dénomination, qui relève du métalangage ordinaire des acteurs et rattache l'existence du genre avant tout à des pratiques d'écriture identifiables pour le parti; ses caractéristiques discursives internes, qui font apparaître l'importance que l'«argumentaire» accorde au dialogisme interdiscursif; son identité de document de communication, en tant qu'il est constitué d'énoncés conçus pour être repris dans le cadre d'un travail d'argumentation mené par le militant; sa multifonctionnalité, alliant affirmation de l'autorité du parti, formation des militants, animation du débat public, et persuasion ; enfin, ses transformations sous l'effet du recours à l'internet dans la communication politique partisane.

We are interested in a kind of text called argumentaire as it exists as a discursive production of contemporary French political parties. Briefly, the argumentaire can be described as a document presenting a particular set of positions that are generally argued and presented as those adopted by the party organization on a particular topic or theme. Five aspects of the argumentaire deserve particular attention: its name, borrowed from the metalanguage of the regular players and connecting the genre to writing practices recognizable by the party; its internal discursive characteristics, which show the importance that the argumentaire gives to the interdiscursive dialogism; its identity of communicative document, insofar as it consists of statements designed to be part of the activist's argumentation; its multifunctionality, combining affirmation of the party's authority, the training of activists, the encouragement of public debate, and persuasion; and finally, its transformations under the effect of Internet use in partisan political communication.

\section{INDEX}

Keywords : argumentaire, dialogism, discourse genre, political discourse, political party Mots-clés : argumentaire, dialogisme interdiscursif, discours politique, genre de discours, parti politique

\section{AUTEUR}

\section{ALICE KRIEG-PLANQUE}

Université Paris-Est Créteil (UPEC), Céditec (EA3119) 